\title{
Teachers at Lock-Down: Generalized Anxiety Disorder and Attitude Towards E-Learning amidst COVID-19 Era
}

\author{
Carlo P. Cortez ${ }^{1 *}$ (1) , Carlo S. Patricio ${ }^{2}$ (), Wilfredo N. Uriarte Jr. ${ }^{2}$ (]) \\ ${ }^{1}$ De La Salle University, PHILIPPINES \\ ${ }^{2}$ STI College Global City, PHILIPPINES \\ *Corresponding Author: carlo222009@yahoo.com \\ Citation: Cortez, C. P., Patricio, C. S., \& Uriarte Jr., W. N. (2021). Teachers at Lock-Down: Generalized Anxiety Disorder and Attitude Towards \\ E-Learning amidst COVID-19 Era. European Journal of Interactive Multimedia and Education, 2(2), e02113. \\ https://doi.org/10.30935/ejimed/11288
}

\begin{abstract}
This study examines the attitude of teachers toward e-learning as the new learning modality and the possible development of general anxiety disorder among teachers amidst COVID-19 pandemic. A sample of 116 teachers were taken before the start of the school year and another 75 teachers after one semester of executing e-learning served as the sample of this study. Data were collected through a self-administered survey questionnaire sent through a google form to comply with the existing lockdown policies of the government. The result indicates that the respondents, in majority, developed mild to severe GAD while preparing and executing e-learning. The data also showed that the acceptability of the teachers were found to be not relatively high nor relatively low. Problems using GAD 7-item questionnaire and TeLRA assessment revealed the rising problem brought by the change in learning delivery system among teachers.

This study examines the attitude of teachers toward e-learning as the new learning modality and the possible development of general anxiety disorder among teachers amidst COVID-19 pandemic. A sample of 116 teachers were taken before the start of the school year and another 75 teachers after one semester of executing e-learning served as the sample of this study. Data were collected through a self-administered survey questionnaire sent through a google form to comply with the existing lockdown policies of the government. The result indicates that the respondents, in majority, developed mild to severe GAD while preparing and executing e-learning. The data also showed that the acceptability of the teachers were found to be not relatively high nor relatively low. Problems using GAD 7-item questionnaire and TeLRA assessment revealed the rising problem brought by the change in learning delivery system among teachers.
\end{abstract}

Keywords: general anxiety disorder, e-learning, e-learning attitudes, mental stress, teachers

Received: 19 May $2021 \bullet$ Accepted: 28 Sep. 2021

\section{INTRODUCTION}

As the COVID-19 pandemic is getting worse, most of the Filipinos are required to stay home as a part of the policies of the community quarantine imposed the by the Philippine government as a measure to prevent further exposure of the citizens to the virus. Most of the establishment are closed and workers are inside their homes waiting for the government to issue updates on when the economy will resume (Official Gazette of the Republic of the Philippines, 2020). Workers worry whether or not they could come back to their jobs amidst the pandemic. This includes the private school teachers whose most status is no work no pay. In the middle of the issue of pandemic and the problem with job compensation, some teachers may experience anxiety that may affect their decision making and perception of different things. Anxiety is a feeling of unease, such as worry or fear that can be mild or severe (NHS, 2018). Teachers' personal and professional lives are consequential for their mental health (Peele \& Wolf, 2020) and the surveillance in the psychological consequences of outbreaks should be monitored (Huang \& Zhao, 2020). In the Philippines, as of 2018, over 3.3 million have to deal with some form of depressive disorder, while around 3.1 million go through some form of anxiety disorder (Rappler, 2019) which would have a possibility of increase due to the ongoing effects of COVID-19. Psychological impact of this pandemic disease on the general population, an increase in depression and stress levels between the first days and the third week of the lockdown has been found (Ozamiz-Etxebarria et al., 2020; Rodríguez-Rey, GarridoHernansaiz, \& Collado, 2020). People with Generalized Anxiety Disorder (GAD) excessively and unrealistically worry for long periods of time over things that may be related to work, personal relationships, etc. and can differ for everyone (Sy, 2019). The GAD-7 is a valid and efficient tool for screening for GAD (Spitzer et al., 2006). GAD-7 is reliable and valid for use among Filipino domestic workers and provides only probable diagnoses that should be confirmed by further evaluation (Hall et.al, 2019; Spitzer et.al, 2006). As the crisis itself creates stress, friction, and anxiety, mental is also increasingly vulnerable and at risk worldwide (Guillermo, 2020). Teacher stress 
which is defined as the experience by a teacher of unpleasant, negative emotions, such as anger, anxiety, tension, frustration or depression, resulting from some aspect of their work as a teacher" Kyriacou (2001) can be a factor in the effectiveness of the teachers' delivery of lesson. It was found that student behavior and the workloads are significant indicators of teachers' anxiety (Mirowsky \& Ross, 2003) which may take an increase due to the new learning modality implemented by schools during the pandemic.

A shift in educational system in the Philippines is about to happen and the teachers, both private and public institutions, are affected. Elearning can occur in or out of the classroom. It can be self-paced, asynchronous learning or may be instructor-led, synchronous learning (Omotayo \& Tiamiyu, 2017). Though it is already being a practice for a while here in the Philippines and outside the country, it is still a challenge for an institution to fully apply this kind learning delivery system especially if the prevailing style is a classroom interaction type of delivery. With the expected shift of educational delivery, a lot of adjustment must be done by the teachers not only on their preparation of teaching materials but likewise with the effectivity of their delivery having a different platform (Garcia, 2017). Educators need to educate themselves to be a good designer, content expert and competent to use technologies in terms of hardware and software to produce effective elearning materials (Hassan et al., 2012). The new trend in educational delivery, if embraced by the educators, will give a positive impact not only in the educational institutions but greatly to the learning and motivation of students. Though, moving learning from classrooms to homes at scale and in a hurry presents enormous challenges, both human and technical (i.e., creating, maintaining, and improving distance learning, or measuring and validating learning) which may affect teachers' delivery effectivity because they need to cope with online education at any level while attending their own personal issues which can cause different stress level among teachers (Komar \& Beo, 2011; UNESCO, 2020c; Wang \& Zhao, 2020). Test of e-learning Related Attitudes (TeLRA) scale that concentrates with themes such as challenges of e-learning, benefits from e-learning, leisure interest on elearning innovations and use of computers are respectively conformed to external variables, teachers' perceived will be used in this study to measure the attitude of teachers towards e-learning (Kisanga \& Ireson, 2016). This research aims to measure the possibility of development of GAD or Generalized Anxiety Disorder among teachers during the time of pandemic in relation with their attitude in e-learning.

\section{METHODOLOGY}

The study is a quantitative method design. The first section of the tool is for the demographics which consist of basic information of the respondents and their material readiness for the e-learning trend. The second section is a standard tool to assess the possibility of the development of General Anxiety Disorder of the respondents which is called GAD-7. For its validity and reliability, please refer to "A Brief Measure for Assessing Generalized Anxiety Disorder" (Spitzer et al., 2006). The third section is a tool measuring the attitude of teachers toward E-Learning called Test of e-Learning Related Attitudes (TeLRA) Scale which was validated through Test of e-Learning Related Attitudes (TeLRA) scale: Development, reliability and validity study (Kisanga \& Ireson, 2016). Our subjects were teachers from different levels with ages ranging from 19 to 54 above. The researchers purposively selected the participants. The researchers of the study also did snowball sampling where requests were made to initial respondents to share the link of the online survey to their co teachers. The study was able to gather a total 116 respondents for the first part of the data gathering and a total 75 responses after six months of applying the new modality of learning delivery. The researchers used Facebook messenger application to instantly send our survey tool to the respondents. The tool was developed through the Google forms platform which contained an introduction of our study, the items and questions to be answered, and the instructions in answering the same. The researchers observed ethical practices in conducting the research. The online survey showed the ethical clause of our data gathering making sure to our participants that the data obtained would only use for the sole purpose of our research. We reminded them that their participation was purely voluntary which means that they may withdraw from answering the survey. We also assured the respondents that their identities and answers would remain confidential.

Data analysis involves chi-square to analyse the relationship of the factors that might affect the development of GAD and their attitude towards e-learning. Factors considered were age, salary, sex, type of educational institution belonged, prior experience in e-learning set up, personal internet connectivity, gadgets owned for e-learning set up, level of students teaching, compensation during lockdown, and years of teaching experience. $T$ test was used to find the difference in the result of the teachers' responses in the gap of six-month period. The data gathered during the gap were independent hence should be comparable. Pearson-r coefficient of correlation was also computed for the results for GAD7 and TeLRA for the two independent set of samples to find the relationship among results.

\section{RESULT}

In the survey conducted to 116 teachers before the 6-month gap and 75 teachers after the 6-month gap with the age range from 19 to above 54 years of age and coming from both from public and private educational institutions, the following were observed:

\section{Teachers' Attitude towards E-Learning}

The researchers gathered two sets of data for the teachers' attitude towards e-learning using TeLRA questionnaire. The first set were gathered before the start of school year 2020-2021 in the month of June. The data from 116 teachers shows (Table 1) the general weighted mean of 2.56 with a standard deviation of 0.35 which is interpreted as an "Agree" with e-learning. It is also notable that the respondents agreed and strongly agreed from the first set of respondents is $55.17 \%$ of the data. After six months, the researchers gathered same data to 75 teachers is showed a 2.62 general weighted mean and standard deviation of 0.39 which implied a little higher level of acceptance but with a higher variance. It was noticeable that $49.33 \%$ of the second set of respondents agreed and strongly agreed with e-learning which is less than the first set. Using student t-test at level of significance of 0.01 , the results from the first and second set of data showed no significant differences in terms of attitude towards e-learning. This is with the tvalue of -1.00029755 and critical value of -2.326 (Table 29). The factors related through Chi-Square such as level teaching in, experience in elearning, monthly salary, gadgets owned, and kind of institution working in showed independence with the result of TeLRA with the level of significance of 0.05 . This result showed both from before and 
Table 1. Summary of Respondent's TeLRA

\begin{tabular}{|c|c|c|c|c|}
\hline TELRA & $\begin{array}{l}\text { BEFORE - } \\
\text { MEAN }\end{array}$ & $\begin{array}{c}\text { BEFORE - } \\
\text { SD } \\
\end{array}$ & $\begin{array}{c}\text { AFTER - } \\
\text { MEAN }\end{array}$ & $\begin{array}{c}\text { AFTER - } \\
\text { SD } \\
\end{array}$ \\
\hline [E-learning is very economical for educational institutions to adopt.] & 2.61 & 0.77 & 2.68 & 0.79 \\
\hline [I believe using e-learning will improve the quality of my work.] & 2.69 & 0.68 & 2.73 & 0.74 \\
\hline [Computers make work more interesting.] & 2.90 & 0.68 & 2.95 & 0.79 \\
\hline [I prefer reading articles in e-learning.] & 2.60 & 0.72 & 2.57 & 0.82 \\
\hline [It is easier to revise electronic educational materials than printed material] & 2.91 & 0.73 & 2.95 & 0.80 \\
\hline [I prefer using a computer to prepare my lessons.] & 2.98 & 0.71 & 3.07 & 0.79 \\
\hline [I feel comfortable reading a text book on a computer screen than a physical text book] & 2.52 & 0.76 & 2.53 & 0.81 \\
\hline [I enjoy teaching using computers.] & 2.65 & 0.74 & 2.73 & 0.86 \\
\hline [Delivering a lecture through electronic technologies is not very difficult.] & 2.51 & 0.80 & 2.44 & 0.87 \\
\hline [E-learning does not requires expensive technical support.] & 2.14 & 0.80 & 2.17 & 0.88 \\
\hline [E-learning does not reduces quality of knowledge attained.] & 2.49 & 0.79 & 2.40 & 0.89 \\
\hline [Interacting with the computer system is not frustrating.] & 2.38 & 0.75 & 2.35 & 0.80 \\
\hline [A face-to-face method is not more learner-centered than E-learning methods.] & 2.01 & 0.79 & 1.96 & 0.78 \\
\hline [I believe using e-learning technologies will improve my job performance.] & 2.66 & 0.69 & 2.85 & 0.73 \\
\hline [Communicating through social networks is fun.] & 2.71 & 0.72 & 2.75 & 0.74 \\
\hline [I like reading magazines on new technology innovations.] & 2.74 & 0.67 & 2.67 & 0.76 \\
\hline [Teaching through e-learning is not tiresome.] & 2.44 & 0.77 & 2.29 & 0.87 \\
\hline [E-learning increases does not learners' social isolation.] & 2.20 & 0.71 & 2.03 & 0.84 \\
\hline [E-learning technologies are not difficult to use.] & 2.53 & 0.72 & 2.71 & 0.78 \\
\hline [Using computer systems does not requires a lot of mental effort.] & 2.28 & 0.78 & 2.32 & 0.86 \\
\hline [Discussions on e-learning technologies are interesting.] & 2.65 & 0.74 & 2.76 & 0.77 \\
\hline [My institution has enough teaching-learning resources to carry out e-learning.] & 2.41 & 0.73 & 2.79 & 0.81 \\
\hline [E-learning will increase teachers' efficiency.] & 2.64 & 0.64 & 2.77 & 0.75 \\
\hline [Working with computers is exciting.] & 2.75 & 0.67 & 2.80 & 0.77 \\
\hline [I like discussing about new e-learning innovations.] & 2.78 & 0.70 & 2.95 & 0.63 \\
\hline [Supporting learners in an e-learning environment is not very difficult.] & 2.19 & 0.71 & 2.27 & 0.89 \\
\hline [E-learning infrastructure is not very expensive for the government to afford.] & 2.09 & 0.78 & 2.27 & 0.81 \\
\hline [It will not be difficult for me to become skilful in the use of e-learning tools.] & 2.68 & 0.72 & 2.68 & 0.77 \\
\hline [I do not make errors frequently when using a Computer.] & 2.66 & 0.67 & 2.72 & 0.69 \\
\hline [Using a computer at home is not very frustrating.] & 2.70 & 0.71 & 2.81 & 0.85 \\
\hline [Using e-learning technologies will allow me to accomplish more work than would otherwise be possible] & 2.78 & 0.67 & 2.95 & 0.66 \\
\hline [I enjoy computer games very much.] & 2.48 & 0.87 & 2.41 & 0.81 \\
\hline [E-learning is not a threat to teachers' employment.] & 2.53 & 0.84 & 2.77 & 0.86 \\
\hline [E-learning will provide me with better learning opportunities than traditional means of learning.] & 2.72 & 0.78 & 2.81 & 0.75 \\
\hline [I find computer online interaction exciting.] & 2.59 & 0.77 & 2.64 & 0.80 \\
\hline [Communicating through electronic mails is not annoying] & 2.74 & 0.77 & 2.75 & 0.81 \\
\hline GENERAL WEIGHTED AVERAGE & 2.56 & 0.35 & 2.62 & 0.39 \\
\hline
\end{tabular}

Table 2. TeLRA vs. Respondent's Level Teaching (Before 6-month Gap)

\begin{tabular}{cccccc}
\hline \multirow{2}{*}{ Level Teaching } & \multicolumn{5}{c}{ TeLRA Result } \\
\cline { 2 - 6 } & Agree & Disagree & Strongly Agree & Strongly Disagree & Grand Total \\
\hline Elementary & 8 & 12 & 1 & 0 & 0 \\
\hline Graduate School & 0 & 2 & 0 & 2 & 2 \\
\hline Junior High School & 27 & 21 & 0 & 0 & 51 \\
\hline Senior High School & 14 & 12 & 0 & 0 & 26 \\
\hline Tertiary & 13 & 3 & 2 & 2 & 16 \\
\hline Grand Total & 62 & 50 & & 116 \\
\hline
\end{tabular}

Table 3. TeLRA vs. Respondent's Level Teaching (After 6-month Gap)

\begin{tabular}{|c|c|c|c|c|c|}
\hline \multirow{2}{*}{ Level Teaching } & \multicolumn{5}{|c|}{ TeLRA Result } \\
\hline & Agree & Disagree & Strongly Agree & Strongly Disagree & Grand Total \\
\hline Elementary & 3 & 4 & 0 & 0 & 7 \\
\hline Graduate School & 8 & 3 & 0 & 0 & 11 \\
\hline Junior High School & 9 & 8 & 2 & 0 & 19 \\
\hline Senior High School & 12 & 22 & 3 & 1 & 38 \\
\hline Tertiary & 32 & 37 & 5 & 1 & 75 \\
\hline Grand Total & 3 & 4 & 0 & 0 & 7 \\
\hline
\end{tabular}


Table 4. TeLRA vs. Respondent's Experience in E-Learning (Before 6-month Gap)

\begin{tabular}{cccccc}
\hline \multirow{2}{*}{ Experience in e-learning } & \multicolumn{5}{c}{ TeLRA Result } \\
\cline { 2 - 6 } & Agree & Disagree & Strongly Agree & Strongly Disagree & Grand Total \\
\hline With Prior Experience & 38 & 23 & 1 & 0 & 62 \\
\hline Without Prior Experience & 24 & 27 & 1 & 2 & 54 \\
\hline Grand Total & 62 & 50 & 2 & 2 & 116 \\
\hline
\end{tabular}

Table 5. TeLRA vs. Respondent's Experience in E-Learning (After 6-month Gap)

\begin{tabular}{cccccc}
\hline \multirow{2}{*}{ Experience in e-learning } & \multicolumn{5}{c}{ TeLRA Result } \\
\cline { 2 - 6 } & Agree & Disagree & Strongly Agree & Strongly Disagree & Grand Total \\
\hline With Prior Experience & 22 & 26 & 5 & 0 & 53 \\
\hline Without Prior Experience & 10 & 11 & 0 & 1 & 22 \\
\hline Grand Total & 32 & 37 & 5 & 1 & 75 \\
\hline
\end{tabular}

Table 6. TeLRA vs. Respondent's Monthly Salary (Before 6-month Gap)

\begin{tabular}{|c|c|c|c|c|c|}
\hline \multirow{2}{*}{ Monthly Salary } & \multicolumn{5}{|c|}{ TeLRA Result } \\
\hline & Agree & Disagree & Strongly Agree & Strongly Disagree & Grand Total \\
\hline 0-9999 & 1 & 2 & 0 & 0 & 3 \\
\hline 10000-19999 & 26 & 16 & 1 & 1 & 44 \\
\hline 20000-29999 & 31 & 21 & 1 & 1 & 54 \\
\hline 30000-39999 & & 1 & 0 & 0 & 1 \\
\hline 40000-49999 & 2 & 2 & 0 & 0 & 4 \\
\hline I prefer not to mention & 2 & 8 & 0 & 0 & 10 \\
\hline
\end{tabular}

Table 7. TeLRA vs. Respondent's Monthly Salary (After 6-month Gap)

\begin{tabular}{ccccccc}
\hline \multirow{2}{*}{ Monthly Salary } & \multicolumn{5}{c}{ TeLRA Result } & \multicolumn{2}{c}{ Agree } \\
\cline { 2 - 6 } & Agree & Disagree & Agree & Strongly Disagree & 0 & 4 \\
\hline $0-9999$ & 4 & 0 & 3 & 1 & 34 \\
\hline $10000-19999$ & 14 & 16 & 2 & 0 & 30 \\
\hline $20000-29999$ & 13 & 15 & 0 & 0 & 1 \\
\hline $30000-39999$ & 1 & 0 & 0 & 0 & 6 \\
\hline I prefer not to mention & 0 & 6 & 5 & 1 & 75 \\
\hline Grand Total & 32 & 37 &
\end{tabular}

Table 8. TeLRA vs. Gadgets Owned (Before 6-month Gap)

\begin{tabular}{cccccc}
\hline \multirow{2}{*}{ Gadgets Owned } & \multicolumn{4}{c}{ TeLRA Result } \\
\cline { 2 - 6 } & Agree & Disagree & Strongly Agree & Strongly Disagree & Grand Total \\
\hline I have exactly 2 electronic gadgets & 41 & 30 & 2 & 0 & 1 \\
\hline I have more than 2 electronic gadgets & 10 & 7 & 0 & 0 & 17 \\
\hline Laptop/Notebook only & 4 & 3 & 0 & 0 & 7 \\
\hline Nothing at all & 1 & 0 & 0 & 1 & 17 \\
\hline Smart Phone alone & 6 & 10 & 2 & 2 & 116 \\
\hline Grand Total & 62 & 50 & & & 0 \\
\hline
\end{tabular}

Table 9. TeLRA vs. Gadgets Owned (After 6-month Gap)

\begin{tabular}{cccccc}
\hline \multirow{2}{*}{ Gadgets Owned } & \multicolumn{5}{c}{ TeLRA Result } \\
\cline { 2 - 6 } & Agree & Disagree & Strongly Agree & Strongly Disagree & Grand Total \\
\hline I have exactly 2 electronic gadgets & 18 & 17 & 2 & 1 & 38 \\
\hline I have more than 2 electronic gadgets & 10 & 11 & 2 & 0 & 23 \\
\hline Laptop/Notebook only & 4 & 8 & 0 & 0 & 2 \\
\hline Smart Phone alone & 0 & 1 & 1 & 1 & 75 \\
\hline Grand Total & 32 & 37 & 5 & \\
\hline
\end{tabular}

after the six-month gap (Tables 25 and 26). One factor, internet connectivity, showed different result from the others. Before the 6month gap, internet connectivity showed independence with the TeLRA result but after the 6-month gap, TeLRA result became dependent with the 75 respondents' perception of the internet connectivity in their area. It is notable that the respondents that they have strong and very strong internet connectivity in their area went from $23.27 \%$ from 116 respondents to $46.67 \%$ of 75 respondents within six months (Tables 10 and 11).

General Anxiety Disorder - 7 Questionnaires (GAD7)

The researchers tried to find the chance of developing general anxiety disorder among teachers amidst the ongoing COVID-19 pandemic. Through the use of GAD7 questionnaire, the researchers 
Table 10. TeLRA vs. Respondents' Internet Connectivity (Before 6-month Gap)

\begin{tabular}{ccccc}
\hline \multirow{2}{*}{ Area Internet Connection } & \multicolumn{2}{c}{ TeLRA Result } \\
\cline { 2 - 5 } & Agree & Disagree & Strongly Agree & Strongly Disagree \\
\hline Fair & 23 & 23 & 1 & 0 \\
\hline Poor & 20 & 20 & 0 & 2 \\
\hline Strong & 14 & 5 & 1 & 0 \\
\hline Very Strong & 5 & 2 & 0 & 0 \\
\hline Grand Total & 62 & 50 & 2 & 2 \\
\hline
\end{tabular}

Table 11. TeLRA vs. Respondents' Internet Connectivity (After 6-month Gap)

\begin{tabular}{ccccc}
\hline \multirow{2}{*}{ Area Internet Connection } & & \multicolumn{2}{c}{ TeLRA Result } \\
\cline { 2 - 5 } & Agree & Disagree & Strongly Agree & Strongly Disagree \\
\hline Fair & 13 & 20 & 0 & 0 \\
\hline Poor & 4 & 3 & 0 & 0 \\
\hline Strong & 14 & 13 & 3 & 1 \\
\hline Very Strong & 1 & 1 & 2 & 0 \\
\hline Grand Total & 32 & 37 & 5 & 1 \\
\hline
\end{tabular}

Table 12. TeLRA vs. Respondents' Institution (Before 6-month Gap)

\begin{tabular}{ccccc}
\hline \multirow{2}{*}{ Kind of Institution } & \multicolumn{3}{c}{ TeLRA Result } \\
\cline { 2 - 5 } & Agree & Disagree & Strongly Agree & Strongly Disagree \\
\hline Private & 27 & 22 & 1 & 1 \\
\hline Public & 35 & 27 & 1 & 1 \\
\hline Grand Total & 62 & 50 & 2 & 2 \\
\hline
\end{tabular}

Table 13. TeLRA vs. Respondents' Institution (After 6-month Gap)

\begin{tabular}{|c|c|c|c|c|c|}
\hline \multirow{2}{*}{ Kind of Institution } & \multicolumn{5}{|c|}{ TeLRA Result } \\
\hline & Agree & Disagree & Strongly Agree & Strongly Disagree & Grand Total \\
\hline Private & 18 & 27 & 4 & 1 & 50 \\
\hline Public & 14 & 10 & 1 & 0 & 25 \\
\hline Grand Total & 32 & 37 & 5 & 1 & 75 \\
\hline
\end{tabular}

Table 14. Summary of Respondent's GAD7 Result

\begin{tabular}{|c|c|c|c|c|}
\hline GAD7 & $\begin{array}{l}\text { BEFORE - } \\
\text { MEAN }\end{array}$ & $\begin{array}{l}\text { BEFORE- } \\
\text { SD }\end{array}$ & $\begin{array}{l}\text { AFTER - } \\
\text { MEAN }\end{array}$ & $\begin{array}{l}\text { AFTER - } \\
\text { SD }\end{array}$ \\
\hline $\begin{array}{l}\text { Over the last } 2 \text { weeks, how often have you been bothered by the following problems? [Feeling nervous, } \\
\text { anxious, or on edge] }\end{array}$ & 1.04 & 0.94 & 1.08 & 0.94 \\
\hline $\begin{array}{l}\text { Over the last } 2 \text { weeks, how often have you been bothered by the following problems? [Not being able to } \\
\text { stop or control worrying] }\end{array}$ & 1.16 & 0.94 & 1.05 & 0.97 \\
\hline $\begin{array}{l}\text { Over the last } 2 \text { weeks, how often have you been bothered by the following problems? [Worrying too much } \\
\text { about different things] }\end{array}$ & 1.18 & 0.92 & 1.16 & 0.94 \\
\hline Over the last 2 weeks, how often have you been bothered by the following problems? [Trouble relaxing] & 1.10 & 0.93 & 0.97 & 0.84 \\
\hline $\begin{array}{l}\text { Over the last } 2 \text { weeks, how often have you been bothered by the following problems? [Being so restless that } \\
\text { it's hard to sit still] }\end{array}$ & 0.88 & 0.92 & 0.93 & 0.88 \\
\hline $\begin{array}{l}\text { Over the last } 2 \text { weeks, how often have you been bothered by the following problems? [Becoming easily } \\
\text { annoyed or irritable] }\end{array}$ & 0.97 & 0.94 & 1.11 & 0.97 \\
\hline $\begin{array}{c}\text { Over the last } 2 \text { weeks, how often have you been bothered by the following problems? [Feeling afraid as if } \\
\text { something awful might happen] }\end{array}$ & 1.20 & 1.02 & 1.05 & 1.00 \\
\hline GENERAL WEIGHTED AVERAGE & 7.53 & 5.45 & 7.36 & 5.59 \\
\hline
\end{tabular}

gathered and tried to measure the development of GAD to the 116 teachers during the month of June 2020 and did the same thing after a 6-month gap. Assessment is based on the result to be interpreted as: 05 points $=$ none; $6-10$ points $=$ mild; $11-15$ points $=$ moderate and $16-$ 21 points $=$ severe (efficacy.org.uk). It was found out that the mean result of GAD7 was 7.53 with standard deviation of 5.45 and 7.36 with standard deviation of 5.59 from before and after the 6-month gap respectively (Table 14). Both result from the 6-month gap was interpreted to be under mild to moderate development of GAD considering their standard deviation. This is also with the fact that $25 \%$ of 116 first set of respondents and $28 \%$ of 75 second set of respondents within the 6-month gap have been identified to have formed a severe GAD during the said period. Student t-test was also used to determine if the difference, though minimal as per Table 14, between the results of GAD among the respondents within the 6-month gap was significant. With the level of significance of 0.01 , critical value of 2.326 and $t$-value of 0.214 , it showed that the responses between the 6-month gap were insignificant (Table 29).

Factors also were tried to relate to the result of GAD7 of the respondents and test its independence among the factors through ChiSquare including their compensation during the lock down period, age, teaching experience, way of doing thing while facing problems, and 
Table 15. GAD7 vs. Respondents' Compensation during Pandemic (Before 6-month Gap)

\begin{tabular}{cccccc}
\hline \multirow{2}{*}{$\begin{array}{c}\text { Compensation Status during } \\
\text { Lockdown }\end{array}$} & Mild & Moderate & None & Severe & Grand Total \\
\cline { 2 - 6 } & 15 & 7 & 16 & 6 & 44 \\
\hline No Work-No Pay & 25 & 10 & 31 & 6 & 72 \\
\hline Regular compensation & 40 & 17 & 47 & 12 & 116 \\
\hline Grand Total & &
\end{tabular}

Table 16. GAD7 vs. Respondents' Compensation during Pandemic (After 6-month Gap)

\begin{tabular}{|c|c|c|c|c|c|}
\hline \multirow{2}{*}{$\begin{array}{c}\text { Compensation Status during } \\
\text { Lockdown }\end{array}$} & \multicolumn{5}{|c|}{ GAD 7 RESULT } \\
\hline & Mild & Moderate & None & Severe & Grand Total \\
\hline No Work-No Pay & 10 & 11 & 15 & 4 & 40 \\
\hline Regular compensation & 13 & 4 & 16 & 2 & 35 \\
\hline Grand Total & 24 & 15 & 31 & 6 & 75 \\
\hline
\end{tabular}

Table 17. GAD7 vs. Respondents' Age (Before 6-month Gap)

\begin{tabular}{|c|c|c|c|c|c|}
\hline \multirow{2}{*}{ Age } & \multicolumn{5}{|c|}{ GAD 7 RESULT } \\
\hline & Mild & Moderate & None & Severe & Grand Total \\
\hline $19-23$ & 6 & 3 & 2 & 3 & 14 \\
\hline $24-28$ & 17 & 7 & 17 & 6 & 47 \\
\hline $29-33$ & 8 & 4 & 10 & 3 & 25 \\
\hline $34-38$ & 4 & 1 & 6 & 0 & 11 \\
\hline $39-43$ & 0 & 0 & 5 & 0 & 5 \\
\hline $44-48$ & 3 & 1 & 3 & 0 & 7 \\
\hline $49-53$ & 1 & & 4 & 0 & 5 \\
\hline 54 and above & 1 & 1 & 0 & 0 & 2 \\
\hline Grand Total & 40 & 17 & 47 & 12 & 116 \\
\hline
\end{tabular}

Table 18. GAD7 vs. Respondents' Age (After 6-month Gap)

\begin{tabular}{cccccc}
\hline Age & \multicolumn{6}{c}{ GAD 7 RESULT } & \multicolumn{2}{c}{ Grand Total } \\
\cline { 2 - 6 } & Mild & Moderate & None & Severe & 7 \\
\hline $19-23$ & 2 & 1 & 4 & 0 & 23 \\
\hline $24-28$ & 10 & 7 & 6 & 2 & 10 \\
\hline $29-33$ & 3 & 1 & 4 & 1 & 9 \\
\hline $34-38$ & 2 & 3 & 3 & 3 & 9 \\
\hline $39-43$ & 2 & 2 & 2 & 0 & 6 \\
\hline $44-48$ & 2 & 0 & 4 & 0 & 10 \\
\hline $49-53$ & 2 & 1 & 7 & 0 & 1 \\
\hline 54 and above & 0 & 0 & 1 & 6 & 75 \\
\hline Grand Total & 24 & 15 & 31 & & \\
\hline
\end{tabular}

Table 19. GAD7 vs. Respondents' Teaching Experience (Before 6-month Gap)

\begin{tabular}{cccccc}
\hline \multirow{2}{*}{ Years in Experience } & \multicolumn{5}{c}{ GAD 7 RESULT } \\
\cline { 2 - 6 } & Mild & Moderate & None & Severe & Grand Total \\
\hline $0-4.9$ years & 25 & 10 & 24 & 1 & 67 \\
\hline $10-14.9$ years & 6 & 2 & 7 & 0 & 16 \\
\hline $15-19.9$ years & 0 & 2 & 0 & 0 & 7 \\
\hline 20 years and above & 1 & 2 & 4 & 3 & 24 \\
\hline $5-9.9$ years & 8 & 1 & 12 & 12 & 116 \\
\hline Grand Total & 40 & 17 & 47 & \\
\hline
\end{tabular}

Table 20. GAD7 vs. Respondents' Teaching Experience (After 6-month Gap)

\begin{tabular}{|c|c|c|c|c|c|}
\hline \multirow{2}{*}{ Years in Experience } & \multicolumn{5}{|c|}{ GAD 7 RESULT } \\
\hline & Mild & Moderate & None & Severe & Grand Total \\
\hline $0-4.9$ years & 12 & 9 & 10 & 2 & 33 \\
\hline $10-14.9$ years & 4 & 2 & 4 & 3 & 13 \\
\hline $15-19.9$ years & 1 & 1 & 6 & 0 & 8 \\
\hline 20 years and above & 0 & 0 & 2 & 0 & 2 \\
\hline $5-9.9$ years & 6 & 3 & 9 & 1 & 19 \\
\hline Grand Total & 24 & 15 & 31 & 6 & 75 \\
\hline
\end{tabular}

gender. The data before the 6-month gap showed that age, gender, and teaching experience have nothing to do with the result of GAD7. But, the result of GAD7 was found to be significantly related to the respondents' compensation during the lock down period and the way 
Table 21. GAD7 vs. Respondents' Coping while Facing Problem (Before 6-month Gap)

\begin{tabular}{|c|c|c|c|c|c|}
\hline \multirow{2}{*}{ Doing Things while Facing Problems } & \multicolumn{5}{|c|}{ GAD 7 RESULT } \\
\hline & Mild & Moderate & None & Severe & Grand Total \\
\hline Not difficult at all & 3 & 0 & 20 & 2 & 25 \\
\hline Somewhat difficult & 33 & 13 & 26 & 3 & 75 \\
\hline Very difficult & 4 & 4 & 1 & 7 & 16 \\
\hline Grand Total & 40 & 17 & 47 & 12 & 116 \\
\hline
\end{tabular}

Table 22. GAD7 vs. Respondents' Coping while Facing Problem (After 6-month Gap)

\begin{tabular}{|c|c|c|c|c|c|}
\hline \multirow{2}{*}{ Doing Things while Facing Problems } & \multicolumn{5}{|c|}{ GAD 7 RESULT } \\
\hline & Mild & Moderate & None & Severe & Grand Total \\
\hline Not difficult at all & 2 & 3 & 13 & 1 & 19 \\
\hline Somewhat difficult & 18 & 10 & 18 & 2 & 48 \\
\hline Very difficult & 3 & 2 & 0 & 3 & 8 \\
\hline Grand Total & 24 & 15 & 31 & 6 & 75 \\
\hline
\end{tabular}

Table 23. GAD7 vs. Respondents' Gender (Before 6-month Gap)

\begin{tabular}{cccccc}
\hline \multirow{2}{*}{ Gender } & \multicolumn{6}{c}{ GAD 7 RESULT } & Severe & Grand Total \\
\cline { 2 - 6 } & Mild & Moderate & None & 29 & 11 \\
\hline Female & 25 & 11 & 1 & 1 & 5 \\
\hline LGBTQ+ & 4 & 0 & 17 & 12 & 35 \\
\hline Male & 11 & 6 & 47 & 116 \\
\hline Grand Total & 40 & 17 &
\end{tabular}

Table 24. GAD7 vs. Respondents' Gender (After 6-month Gap)

\begin{tabular}{|c|c|c|c|c|c|}
\hline \multirow{2}{*}{ Gender } & \multicolumn{5}{|c|}{ GAD 7 RESULT } \\
\hline & Mild & Moderate & None & Severe & Grand Total \\
\hline Female & 13 & 8 & 15 & 3 & 39 \\
\hline LGBTQ+ & 0 & 1 & 0 & 0 & 1 \\
\hline Male & 10 & 6 & 16 & 3 & 35 \\
\hline Grand Total & 24 & 15 & 31 & 6 & 75 \\
\hline
\end{tabular}

Table 25. Summary of Chi-Square Result for TeLRA (Before 6-Month Gap)

\begin{tabular}{cccccc}
\hline \multicolumn{7}{c}{ Summary of Chi Square Result - Before 6-Month Period } \\
\hline \multicolumn{7}{c}{ Factors vs. TeLRA } \\
\hline Factors & Chi Square & Critical Value & Degree of Freedom & LS & Remarks \\
\hline Level Teaching & 13.52 & 21.03 & 12 & 0.05 & Independent \\
\hline Experience in e-Learning & 4.95 & 7.815 & 3 & 0.05 & Independent \\
\hline Monthly Salary & 9.09 & 25 & 15 & 0.05 & Independent \\
\hline Gadgets Owned & 6.48 & 21.03 & 12 & 0.05 & Independent \\
\hline Internet Connectivity & 9.99 & 16.92 & 9 & 0.05 & Independent \\
\hline Kind of Institution & 0.10 & 7.815 & 3 & 0.05 & Independent \\
\hline
\end{tabular}

Table 26. Summary of Chi-Square Result for TeLRA (After 6-Month Gap)

\begin{tabular}{|c|c|c|c|c|c|}
\hline \multicolumn{6}{|c|}{ Summary of Chi Square Result - Before 6-Month Period } \\
\hline & & Factors & RA & & \\
\hline Factors & Chi Square & Critical Value & Degree of Freedom & LS & Remarks \\
\hline Level Teaching & 8.14 & 21.03 & 12 & 0.05 & Independent \\
\hline Experience in e-Learning & 4.54 & 7.815 & 3 & 0.05 & Independent \\
\hline Monthly Salary & 14.24 & 21.03 & 12 & 0.05 & Independent \\
\hline Gadgets Owned & 9.86 & 16.92 & 9 & 0.05 & Independent \\
\hline Internet Connectivity & 18.27 & 16.92 & 9 & 0.05 & Dependent \\
\hline Kind of Institution & 3.12 & 7.815 & 3 & 0.05 & Independent \\
\hline
\end{tabular}

they do thing while facing problems. There are $37.93 \%$ of 116 respondents (Table 27) from this period were in "no work, no pay" status and $64.66 \%$ (Table 16) were saying it is somewhat difficult for them to accomplish things while facing problems. The data gathered after the 6-month gap showed almost the same result. All the factors showed independence with the result of GAD7 except for "the way they do things while facing problems" which showed significant relationship with the result of GAD7. There are about $64 \%$ of 75 respondents after the 6-month gap said that it is somewhat difficult for them to accomplish task while facing problems. Pearson-r coefficient of correlation was also use to describe the degree of relationship between the result of TeLRA and GAD7 from before and after the 6-month gap it showed a weak negative correlation for both set of data (Table 30). 
Table 27. GAD7 vs. Respondents' Compensation during Pandemic (Before 6-month Gap)

\begin{tabular}{|c|c|c|c|c|c|}
\hline \multicolumn{6}{|c|}{ Summary of Chi Square Result - Before 6-Month Period } \\
\hline \multicolumn{6}{|c|}{ Factors vs. GAD7 } \\
\hline Factors & Chi Square & Critical Value & Degree of Freedom & LS & Remarks \\
\hline Compensation during Lockdown & 54.60 & 7.815 & 3 & 0.05 & Dependent \\
\hline Age & 21.91 & 32.671 & 21 & 0.05 & Independent \\
\hline Teaching Experience & 18.14 & 21.026 & 12 & 0.05 & Independent \\
\hline Doing Things while Facing Problems & 46.45 & 12.592 & 6 & 0.05 & Dependent \\
\hline Gender & 8.89 & 12.592 & 6 & 0.05 & Independent \\
\hline
\end{tabular}

Table 28. GAD7 vs. Respondents' Compensation during Pandemic (After 6-month Gap)

\begin{tabular}{|c|c|c|c|c|c|}
\hline \multicolumn{6}{|c|}{ Summary of Chi Square Result - Before 6-Month Period } \\
\hline & & Factor & & & \\
\hline Factors & Chi Square & Critical Value & Degree of Freedom & LS & Remarks \\
\hline Compensation during Lockdown & 4.05 & 7.815 & 3 & 0.05 & Dependent \\
\hline Age & 26.18 & 32.671 & 21 & 0.05 & Independent \\
\hline Teaching Experience & 13.77 & 21.026 & 12 & 0.05 & Independent \\
\hline Doing Things while Facing Problems & 20.16 & 12.592 & 6 & 0.05 & Dependent \\
\hline Gender & 4.57 & 12.592 & 6 & 0.05 & Independent \\
\hline
\end{tabular}

Table 29. T-Test (Before and After) 6-month Gap

\begin{tabular}{ccc}
\hline & T-Test (Before and After) 6-month Gap & GAD7 \\
\hline Values & TeLRA & 2.326 \\
\hline Critical Value & -2.326 & 0.01 \\
\hline Level of Significance & 0.01 & 0.214016396 \\
\hline T-Value & -1.000297553 & 189 \\
\hline Degree of Freedom & 189 & Non Significant \\
\hline Interpretation & Non Significant & \\
\hline
\end{tabular}

Table 30. TeLRA vs. GAD7 Relationship

\begin{tabular}{cccc}
\hline & TeLRA vs. GAD7 Relationship & \\
\hline Period & Pearson-r & Sample & Interpretation \\
\hline Before 6-month Gap & -0.25019806 & 116 & Weak Negative Correlation \\
\hline After 6-month Gap & -0.29762542 & 75 & Weak Negative Correlation \\
\hline T-test correlation significance & $\mathrm{T}=-3.67 ; \mathrm{df}=2.457$ & 191 & Significant \\
\hline
\end{tabular}

\section{DISCUSSION}

The discussion in this research will focus on teachers' acceptability of the new learning modality being implemented in the Philippine educational system which is e-learning and the possibility of teachers developing General Anxiety Disorder due to some factors and the COVID-19 pandemic.

\section{Teachers' E-Learning Related Attitudes (TeLRA) and Some External Factors}

E-learning is not totally new in the Philippine educational system for some of the schools are applying blended type of learning way before the 2020 pandemic. But, this the first time that it is fully implemented due to the elimination of a face to face interaction between teachers and students. On the other hand, global school closures are impacting over $60 \%$ of the world's student population, and in several countries, the implemented localized closures could impact millions of additional learners (UNESCO, 2020b). It is an issue whether teachers' degree of

acceptability in the full implementation of electronic learning among students. Some teachers may not be confident in implementing new modalities which would stress the importance of training for the teacher (Beilock \& Willingham, 2014) before the sudden implementation. In the conversion of some part of TeLRA to gain the actual weighted mean, the negative statements' interpretation has to be reversed. The result, in general, for the first and second set of data shows that the respondents do Agree with e-learning with general weighted average of 2.56 and 2.62 for both set of data. There were noticeable questions in the TeLRA questionnaire that is different from the overall result. It should be noted that result of the TeLRA falls not far from the border line of interpretation of Likert scale so it is understandable that there will be items that is below the general result that will create different interpretation. TeLRA items 10, 11, 12, 13, 17, $18,20,26,27$ that were stated negatively had the results converted inversely to fit the computation for weighted mean were all resulted to a weighted mean of below 2.50 which is interpreted as "disagree". This means that before the results were computed, the statements were converted into positive. Item number 22 and 32, positively stated items, also recorded a low weighted mean. The following are the items that showed a negative response from the TeLRA:

- E-learning do not require expensive technical support

- E-learning does not reduce quality of knowledge attained

- Interacting with the computer system is not frustrating

- A face-to-face method is not more learner-centered than Elearning methods

- Teaching through e-learning is not tiresome

- E-learning does not increase learners' social isolation

- Using computer systems does not require a lot of mental effort 
- Supporting learners in an e-learning environment is not very difficult

- E-learning infrastructure is not very expensive for the government to afford

- I enjoy computer games very much

- My institution has enough teaching-learning resources to carry out e-learning

Teachers are concerned with the cost of implementing e-learning including the execution and usage of the electronic devices involved in the modality. It also indicates that teachers were not confident to the effectivity the new modality as well as its effect to the students. As also mentioned in Education Youth Policy Analysis Unit in the Education Audiovisual Culture Executive Agency (2020) the work of teachers become even more difficult when this direct contact must be replaced by an online relationship. Creation of better learning materials (Hassan et al., 2012) and familiarity in the e-learning platform (Garcia, 2017) were indicated as part of the concerns of the teachers. Internet connectivity was the only factor manifested a different result in the 6month gap. It only showed that area internet connectivity of the teachers really affects the teachers' acceptability of an online modality. On the side of the teachers, majority agreed that using computer system require a lot of mental effort considering that most of them also agreed that their institutions have no enough teaching-learning resources to carry out e-learning (Table 1). This was supported by Mailazar et al. (2020) where they emphasized that the most significant barriers for mathematics teachers are internet connectivity and lack of access to electronic gadgets which likewise resulted in the respondent's agreement that interacting with the computer system is somehow frustrating.

\section{Teachers and General Anxiety Disorder}

Teachers, similar to other peoples' situation during the time of pandemic, are subject to possible mental conditions given some work related and personal problems they may encounter. Worry, panic, anxiety, or depression-related distress (Bao et al., 2020) can appear more commonly among people nowadays. Transitions to distance learning platforms tend to be messy and frustrating, even in the best circumstances (UNESCO, 2020c). Performance of the teachers during the sudden shift of educational delivery may decline due to mental stress the crisis can bring. Debilitating performance anxiety is defined as “...strong but delimited fears that severely compromise an individual's capacity to execute a task at a level that could be reasonable expected, which is crucial to that person's normal adjustment" (Powell, 2004). Though outcome of a GAD7 assessment is just an recommendation for the subjects, it should be noted that teachers assessed before and after the 6-month gap of this study were assessed to be mild to moderate considering their standard deviation. There are about $59.48 \%$ and $60 \%$ of the respondents from before and after the 6-month gap were assessed to possibly have from mild to severe general anxiety disorder during the time when lock downs and shut downs of different companies where ongoing. Most of the factors related to the GAD7 results in this study were mostly insignificant aside from the manner of compensation during the lock down period and the way teacher deals with work when having problems which showed dependence with the GAD7 result. There are $63.63 \%$ and $62.5 \%$ from the respondents with no work no pay status before and after the 6-month gap were assessed to be in mild to severe GAD (Tables 27 and 28). It was $78.45 \%$ and $74.66 \%$ of the respondents from the 6-month gap found difficulty doing work while dealing with problems where $70.33 \%$ and $67.86 \%$ respectively were assessed to be from mild to severe (Tables 15 and 16). In the present situation where students likely to develop anxiety from mild to severe level (Sundarasen et al., 2020), teachers as well suffer the same mental pressure as they attend the same level of competency despite the sudden change in modality. The odds of anxiety were higher among the female students compared to that among the male students (Sundarasen et al., 2020) which also manifested in this study where female teachers have higher chance to develop mild to severe GAD (Tables 23 and 24). Teachers need, indeed, socio-emotional support to face the extra pressure being put on them to deliver learning in a time of crisis (UNESCO, 2020d). In order for the new modality to be effective, in addition to the well-prepared facilities, teachers' mental health should also be addressed during the times of crisis. Moreover, providing support for teachers own psychosocial well-being is an essential component of supporting students (Inter-Agency Standing Committee, 2007). It is also notable that $64.18 \%$ and $69.7 \%$ from the respondents with less than 5 years teaching experience from before and after the 6month gap, respectively, were assessed to have developed mild to severe GAD. Klassen and Chiu (2010) also found that there is a relationship between the years of experience and challenges academics face on the job.

\section{TeLRA vs. GAD7}

Teachers' mental health and e-learning capabilities are two of the essential factors to consider during the full implementation of elearning for all basic, secondary, and tertiary education in order for the delivery to be effective one way or another. In this study, we utilized TeLRA to measure teachers' acceptability of e-learning though its predictive validity has not yet examined despite its internal validity (Kisanga \& Ireson, 2016). General Anxiety Disorder 7-item questionnaires were also utilized to assess the possibility of development of GAD to the teachers during the time of pandemic. The Generalized Anxiety Disorder-7 (GAD-7) was developed in the USA as a valuable screening tool for detecting GAD in primary care patients (Spitzer et al., 2006). For many teachers, there remains shame in discussing stress with one's peers and even with one's primary health care provider (Ferguson et al., 2007). It is about time to discuss the possibility of teachers acquiring mental disorder specially in the time crisis such as COVID-19 pandemic. Teachers' effectivity in an unfamiliar platform using electronic gadgets and internet may decline per delivery of intended learning outcome. Likewise, due to uncertainty of the days during the lockdown, anxiety may develop to some of people specially the teachers. The data gathered from the two sets of respondents in the 6-month gap shows a weak negative correlation which indicates an inverse relationship between the GAD7 and TeLRA results. This means that as one variable gets higher, the other goes down. T-test also showed, though weak, that the relationship between the two variables is significant. This means that there exist a non-zero correlation between the result of GAD7 and TeLRA. Addressing the problem with the teachers' e-learning acceptability may decrease the possibility of acquiring GAD among teachers.

\section{CONCLUSION}

Teachers, in the varying needs of educational system in time of crisis, encounters a lot of frustration in delivering e-learning modality. 
Frustrations and stress brought pandemic and new learning delivery system may affect the effectivity of teachers. In this study, the researchers used 116 teachers as respondents before the beginning of the school year and another 75 teachers after a semester to examine the variability of the responses within the mentioned time. With the analysis of the data gathered, the following conclusion were found:

- Teachers, as of the moment, are still having a hard time adjusting in the new normal of teaching after more than a semester of undergoing and implementing the new teaching modality. This is despite having borderline general weighted mean 2.56 and 2.62 which interpret as "Agree" in the Likert scale.

- The items showing low result in the TeLRA indicates the concerns of the teachers such as cost of implementation, effects in the quality of teaching, frustrations during lectures, social isolation for both teachers and students, mental stress during online class, government and the local school's capability in sustaining and implementing e-learning. The research also showed that teacher's acceptability of e-learning was dependent of the internet connectivity of either their institution or their own home.

- Factors such as level of teaching, experience in e-learning, monthly salary, gadgets owned, and type of institution were found to be independent in the result of TeLRA which means that these factors have not affected the respondents' perception of e-learning.

- The teachers, in average, were assessed to be in the stage of mild to moderate GAD with a general weighted average of 7.53 and 7.36 respective of before and after the 6-month gap. It is notable that majority of the teachers were assessed to be between mild to severe. It was found out that female assessed by GAD7 to have higher probability of developing mild to severe GAD with chance of $70.33 \%$ and $67.86 \%$ from before and after the 6 month gap, respectively.

- Higher probability of acquiring mild to severe GAD also manifested to the respondents with under 5-year experience showing a chance of $64.18 \%$ and 69.7 from both before and after the 6-month gap respectively.

- GAD7 result showed dependence to the compensation status of the respondents before the 6-month gap. It goes to say that before the opening of classes, the compensation status of the teachers contributed to the result of GAD7 assessment.

- This study showed as well that most teachers tend to have difficulty doing work while facing problems. It is equivalent to $78.45 \%$ and $74.66 \%$ of the respondents from the 6-month gap. This also shows that teachers have the same chance of experiencing difficulty in delivering learning modality while facing own personal problem.

- With the screening did by the GAD7, $59 \%$ to $60 \%$ of the teachers tend to develop mild to severe GAD. Just like the students in which schools considering their mental health, teacher might as well be given considered in reducing the level of stress brought by work related endeavors. As showed by the data, teachers find some difficulty in dealing with workloads when having problems that affects them mentally.
- There is a weak negative correlation between the results of GAD7 and TeLRA among teachers involved before and after the 6-month gap. This shows that teachers can have a lower GAD7 assessment while having a high TeLRA scale score. Significant relationship also manifested, though weak, between the two values. This indicates that relationship between GAD7 and TeLRA results must be taken into consideration in creating adjustments on teacher's workloads and training in order for them to be more effective. Working on a higher TeLRA for teachers can result to a better GAD7 which can improve teachers' manner of dealing workloads while facing problems.

Author contributions: All authors were involved in concept, design, collection of data, interpretation, writing, and critically revising the article. All authors approve final version of the article.

Funding: The authors received no financial support for the research and/or authorship of this article.

Declaration of interest: Authors declare no competing interest.

Data availability: Data generated or analysed during this study are available from the authors on request.

\section{REFERENCES}

Aslrasouli, M., \& Vahid, M. S. P. (2014). An investigation of teaching anxiety among novice and experienced Iranian EFL teachers across gender. Procedia - Social and Behavioral Sciences, 98, 304-313. https://doi.org/10.1016/j.sbspro.2014.03.421

Bao, Y., Sun, Y., Meng, S., Shi, J., and Lu, L. (2020). 2019-nCoV epidemic: address mental health care to empower society. Lancet, 22, e37-e38. https://doi.org/10.1016/S0140-6736(20)30309-3

Beilock, S. L., \& Willingham, D. T. (2014). Mathematics anxiety: Can teachers help students reduce it? American Educator, 38(2), 28-32.

Bowling, N. A., Alarcon, G. M., Bragg, C. B., \& Hartman, M. J. (2015). A meta-analytic examination of the potential correlates and consequences of workload. Work \& Stress, 29(2), 95-113. https://doi.org/10.1080/02678373.2015.1033037

Education Youth Policy Analysis Unit in the Education Audiovisual Culture Executive Agency (2020). Initial education for teachers working in early childhood and school education. https://eacea.ec. europa.eu/national-policies/eurydice/content/initial-educationteachers-working-early-childhood-and-school-education-78_en

Ferguson, K., Frost, L., Kirkwood, K., \& Hall, D. (2007). Teacher stress and stigma in northern Ontario. In J. A. LeClair, \& L.T. Foster (Eds.), Contemporary issues in mental health: Concepts, policy, and practice (pp. 211-225). Western Geographical Press.

Garcia, M. (2017). E-learning technology adoption in the Philippines: An investigation of factors affecting Filipino college students' acceptance of learning management systems. The International Journal of E-Learning and Educational Technologies in the Digital Media (IJEETDM), 3(3), 118-130. https://doi.org/10.17781/P002374

Hall, B. J., Garabiles, M. R., \& Latkin, C. A. (2019). Work life, relationship, and policy determinants of health and well-being among Filipino domestic Workers in China: a qualitative study. BMC Public Health, 19, 229. https://doi.org/10.1186/s12889-0196552-4 
Hassan H., Hassan F., Dahalan N., Zuraini Zakaria Z., Nor W.A. (2012). Evaluating Mathematics e-learning materials: Do Evaluators agree with distance learners? Procedia - Social and Behavioral Sciences, 67, 189-195. https://doi.org/10.1016/j.sbspro.2012.11.320

Huang, Y., \& Zhao, N. (2020). Generalized anxiety disorder, depressive symptoms and sleep quality during COVID-19 outbreak in China: a web-based cross-sectional survey. Psychiatry Research, 288, 112954 https://doi.org/10.1016/j.psychres.2020.112954 Erratum in: Psychiatry Research, 299, 113803.

Inter-Agency Standing Committee (2007b). Action Sheet 7.1 Strengthen access to safe and supportive education. In IASC guidelines on mental health and psychosocial support in emergency settings. https://interagencystandingcommittee.org/system/files/ legacy_files/guidelines_iasc_mental_health_psychosocial_june_20 07.pdf

Kisanga, D., \& Ireson, G. (2016). Test of e-learning related attitudes (TeLRA) scale: Development, reliability and validity study. International Journal of Education and Development using ICT, 12(1). https://www.learntechlib.org/p/173442/

Klassen, R. M., \& Chiu, M. M. (2010). Effects on teachers' self-efficacy and job satisfaction: Teacher gender, years of experience, and job stress. Journal of Educational Psychology, 102(3), 741-756. https://doi.org/10.1037/a0019237

Kyriacou, C. (2001). Teacher stress: Directions for future research. Educational Review, 53(1), 27-35. https://doi.org/10.1080/ 00131910120033628

Mirowsky, J., \& Ross, C.E. (2003). Social causes of psychological distress (2nd ed). Aldine De Gruyther.

National Health Service (2018). Overview - Generalised anxiety disorder in adults. https://www.nhs.uk/mental-health/conditions/generalised -anxiety-disorder/overview/

Official Gazette of the Republic of the Philippines. (2020). Omnibus guidelines on the implementation of community quarantine in the Philippines. https://www.officialgazette.gov.ph/downloads/2020/ 05may/20200522-omnibus-guidelines-on-the-implementationof-community-quarantine-in-the-philippines

Omotayo, F. O., \& Tiamiyu, W. A. (2017). Influence of socioenvironmental forces on use of e-learning by teachers in selected tertiary institutions in Oyo state, Nigeria. The African Journal of Information Systems, 9(1), 1.
Ozamiz-Etxebarria, N., Idoiaga Mondragon, N., Dosil Santamaría, M., and Picaza Gorrotxategi, M. (2020). Psychological symptoms during the two stages of lockdown in response to the COVID-19 outbreak: an investigation in a sample of citizens in northern Spain. Frontiers in Psychology, 11, 1491. https://doi.org/10.3389/ fpsyg.2020.01491

Peele, M., \& Wolf, S. (2020). Predictors of anxiety and depressive symptoms among teachers in Ghana: Evidence from a randomized controlled trial. Social Science \& Medicine, 253, 112957. https://doi.org/10.1016/j.socscimed.2020.112957

Powell, D. (2004, August). Treating individuals with debilitating performance anxiety: An introduction. Journal of Clinical Psychology, 60(8), 801-809. https://doi.org/10.1002/jclp.20038

Rappler (2019). Battling depression and anxiety. https://www.rappler.com/video/battling-depression-anxiety

Rodríguez-Rey, R., Garrido-Hernansaiz, H., \& Collado, S. (2020). Psychological impact of COVID-19 in Spain: Early data report. Psychological Trauma: Theory, Research, Practice, and Policy, 12(5), 550-552. https://doi.org/10.1037/tra0000943

Spitzer, R. L., Kroenke, K., Williams, J. B. W., \& Löwe, B. (2006). A brief measure for assessing generalized anxiety disorder: The GAD7. Archives of Internal Medicine, 166(10), 1092-1097. https://doi.org/10.1001/archinte.166.10.1092

UNESCO (2020a). Education: From disruption to recovery. https://en.unesco.org/COVID19/educationresponse/

UNESCO (2020b). Adverse consequences of school closures. https:// en.unesco.org/COVID19/educationresponse/consequences

UNESCO (2020c). Adverse consequences of school closures. https://en.unesco.org/covid19/educationresponse/consequences

UNESCO (2020d). COVID-19 webinar: A new World for teachers, education's frontline workers - COVID-19 education webinar \#2. https://en.unesco.org/news/covid-19-webinar-new-worldteachers-educations-frontline-workers-covid-19-educationwebinar-2

Wang, C., \& Zhao, H. (2020). The Impact of COVID-19 on Anxiety in Chinese University Students. Frontiers in Psychology, 11, 1168. https://doi.org/10.3389/fpsyg.2020.01168 